\title{
Clinical Significance of Esophageal Hypertensive Peristaltic Contractions on High-resolution Manometry
} (Neurogastroenterol Motil 2015;27:229-236)

Jung Ho Park

Department of Internal Medicine, Kangbuk Samsung Hospital, Sungkyunkwan University College of Medicine, Seoul, Korea

\section{Summary}

Esophageal peristalsis consists of a chain of relaxing sphincters and contracting segments. First, the segment of skeletal muscle (S1) contracts, then the proximal segment of smooth muscle (S2), and finally the distal segment of smooth muscle (S3). ${ }^{1}$ The esophageal hypercontractility disorder has been postulated to be caused by imbalance between esophageal contraction and relaxation. A variant of this disorder, where contraction amplitudes of S3 are disproportionately exaggerated compared with those of S2, can produce esophageal symptoms. ${ }^{2}$ However, some of these contraction patterns could be averaged out and may not be registered as abnormal. Therefore, Mello et $\mathrm{al}^{3}$ tried to determine the clinical significance of these contraction patterns and the diagnostic sensitivity of Chicago classification for these patterns.

The authors found that merged segments were in $5.6 \%$, and exaggerated S3 in another $12.5 \%$, but only $17-50 \%$ had a Chicago classification diagnosis. The cohorts with merged segments and exaggerated S3 had significantly higher proportions of abnormal relaxation of esophageal body during multiple rapid swallowing (MRS) $(P<0.005$ for each comparison) and presenting symptoms (chest pain and dysphagia, $P=0.040$ ) than healthy controls. The authors concluded that merged segments and exaggerated S3 may represent esophageal hypercontractility disorder from abnormal relaxation and/or contraction, and the Chicago classification for these contraction patterns may not be sensitive.

\section{Comments}

The clinical relevance of esophageal hypercontractility disorders is still debated because the symptoms are not correlated with the amplitude of esophageal peristalsis. ${ }^{4}$ It is also controversial whether nutcracker esophagus is a true esophageal motor disorder or only a manometric marker in non-cardiac chest pain. $^{5}$

This study showed that merged segments and exaggerated S3 in high-resolution manometry (HRM) could be encountered in symptomatic patients, but also be seen even in healthy controls

Received: June 17, 2015 Revised: None Accepted: June 30, 2015

(c) This is an Open Access article distributed under the terms of the Creative Commons Attribution Non-Commercial License (http://creativecommons. org/licenses/by-nc/4.0) which permits unrestricted non-commercial use, distribution, and reproduction in any medium, provided the original work is properly cited.

${ }^{*}$ Correspondence: Jung Ho Park, MD Department of Internal Medicine, Kangbuk Samsung Hospital, Sungkyunkwan University School of Medicine, 29, Saemunan-ro, Jongno-gu, Seoul 03181, Korea Tel: +82-2-2001-2059, Fax: +82-2-2001-2485, E-mail: pjho3@hotmail.com

Financial support: None.

Conflicts of interest: None.

ORCID: Jung Ho Park, http://orcid.org/0000-0003-2568-128X. 
at low rate. However, these contraction patterns in HRM can show up in very heterogeneous group of patients. Depending on the results of distal contractile integral response (DCI) to multiple rapid swallowing, DCIs of some merged segments and exaggerated $\mathrm{S} 3$ were over $5000 \mathrm{mmHg} \cdot \mathrm{cm} \cdot \mathrm{sec}$. Furthermore, about $18 \%(6 / 34)$ of exaggerated S3 was over $8000 \mathrm{mmHg} \cdot \mathrm{cm} \cdot \mathrm{sec}$. It means that quite a number of patients with merged segments and exaggerated S3 in HRM could be diagnosed as "nutcracker esophagus" and "Jackhammer esophagus," and these patients could be more likely to have symptoms such as heartburn, chest pain, and dysphagia, ${ }^{6,7}$ than those with low DCI and merged segments and exaggerated $\mathrm{S} 3$. As a result, these contraction patterns in HRM, low DCI and merged esophageal smooth muscle segments and exaggerated S3 do not seem to represent as clinically significant HRM findings.

Recently, the significance of hypertensive peristaltic contractions (DCI 5000-8000 $\mathrm{mmHg} \cdot \mathrm{cm} \cdot \mathrm{sec}$ ) was questioned and the designation of "hypertensive peristalsis" was eliminated in Chicago classification version $3.0 .^{8}$ Also, jackhammer esophagus was redefined as the occurrence of $20 \%$ of swallows, instead of "at least one swallow," with a DCI $>8000 \mathrm{mmHg} \cdot \mathrm{cm} \cdot \mathrm{sec}$ and normal latency. The investigation into the better criteria for esophageal hypertensive peristaltic contractions is still in progress.

\section{References}

1. Clouse RE, Staiano A. Topography of normal and high-amplitude esophageal peristalsis. Am J Physiol 1993;265(6 Pt 1):G1098-G1107.

2. Kushnir VM, Prakash Gyawali C. High resolution manometry patterns distinguish acid sensitivity in non-cardiac chest pain. Neurogastroenterol Motil 2011;23:1066-1072.

3. Mello MD, Duraiswamy S, Price LH, Li Y, Patel A, Gyawali CP. Exaggerated smooth muscle contraction segments on esophageal high-resolution manometry: prevalence and clinical relevance. Neurogastroenterol Motil 2015;27:229-236.

4. Tsuboi K, Mittal SK, Legner A, Yano F, Filipi CJ. Relationship between manometric findings and reported symptoms in nutcracker esophagus: insights gained from a review of 313 patients. J Gastroenterol 2010;45:1033-1038.

5. Kahrilas PJ. Nutcracker esophagus: an idea whose time has gone? Am J Gastroenterol 1993;88:167-169.

6. Lufrano R, Heckman MG, Diehl N, DeVault KR, Achem SR. Nutcracker esophagus: demographic, clinical features, and esophageal tests in 115 patients. Dis Esophagus 2015;28:11-18.

7. Crespin O, Tatum R, Yates R, et al. Esophageal hypermotility: cause or effect? Dis Esophagus Online First: 20 Apr 2015. doi: 10.1111/ dote. 12367.

8. Kahrilas PJ, Bredenoord AJ, Fox M, et al. The Chicago Classification of esophageal motility disorders, v3.0. Neurogastroenterol Motil 2015;27:160-174. 